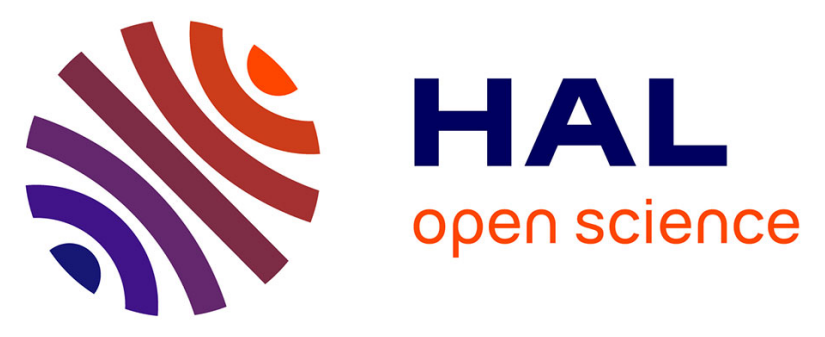

\title{
Nonlinear control of a nano-hexacopter carrying a manipulator arm
}

Jonatan Álvarez-Muñoz, Nicolas Marchand, Fermi Guerrero-Castellanos, A. E. Lopez-Luna, Jose Juan Téllez-Guzmán, Jose Colmenares-Vazquez, Sylvain Durand, Jonathan Dumon, Ghatfan Hasan

\section{To cite this version:}

Jonatan Álvarez-Muñoz, Nicolas Marchand, Fermi Guerrero-Castellanos, A. E. Lopez-Luna, Jose Juan Téllez-Guzmán, et al.. Nonlinear control of a nano-hexacopter carrying a manipulator arm. IROS 2015 - IEEE/RSJ International Conference on Intelligent Robots and Systems, Sep 2015, Hamburg, Germany. hal-01211118

\section{HAL Id: hal-01211118 \\ https://hal.science/hal-01211118}

Submitted on 27 Jan 2016

HAL is a multi-disciplinary open access archive for the deposit and dissemination of scientific research documents, whether they are published or not. The documents may come from teaching and research institutions in France or abroad, or from public or private research centers.
L'archive ouverte pluridisciplinaire HAL, est destinée au dépôt et à la diffusion de documents scientifiques de niveau recherche, publiés ou non, émanant des établissements d'enseignement et de recherche français ou étrangers, des laboratoires publics ou privés.

\section{(1) (1) $\$$}

Distributed under a Creative Commons Attribution - NonCommercial - NoDerivatives| 4.0 


\title{
Nonlinear control of a nano-hexacopter carrying a manipulator arm
}

\author{
J. U. Alvarez-Muñoz ${ }^{1}$, N. Marchand ${ }^{1,2}$, J. F. Guerrero-Castellanos ${ }^{3}$, A. E. López-Luna ${ }^{3}$, J. J. Téllez-Guzmán ${ }^{1}$, \\ J. Colmenares-Vazquez ${ }^{1}$, S. Durand ${ }^{4}$, J. Dumon ${ }^{1}$ and G. Hasan ${ }^{1}$
}

\begin{abstract}
This paper proposes a simple solution for stabilization of a nano-hexacopter carrying a manipulator arm in order to increase the type of missions achievable by these types of systems. The manipulator arm is attached to the lower part of the hexacopter. The motion of the arm induces a change of the center of mass of the whole body, which induces torques which can produce the loss of stability. The present work deals with the stabilization of the whole system - that is hexacopter and arm - by means of a set of nonlinear control laws. First, an attitude control, stabilizes the hexacopter to a desired attitude taking into account the movement of the arm. Then, a suitable virtual control and the translational dynamics allow the formulation of a nonlinear controller, which drives the aerial vehicle to a desired position. Both controls consist in saturation functions. Experimental results validate the proposed control strategy and compares the results when the motion of the arm is taken into account or not.
\end{abstract}

\section{INTRODUCTION}

Aerial manipulation has been an active area of research in recent times, mainly because the active tasking of Unmanned Aerial Vehicles (UAVs) increases the employability of these vehicles for various applications. For active tasking one would consider manipulation, grasping and transporting etc. Unlike fixed wings UAVs, that are incapable of driving their velocity to zero, VTOL (Vertical Take-Off and Landing) vehicles with four, six, or eight rotary wings (afterwards called multirotors) are ideally suited to the task of aerial manipulation or grasping. However, there are many challenges in aerial grasping for these vehicles. The biggest challenge arises from their limited payload. While multiple robots can carry payloads with grippers [1] or with cables [2], their end effectors and grippers have to be light weight themselves and capable of grasping complex shapes. Secondly, the dynamics of the robot is significantly altered by the addition of payloads. This is indeed an attraction in assembly because aerial robots can use this principle to sense disturbance forces and moments. However, for payload transport, it is necessary that the robots are able to

\footnotetext{
${ }^{1}$ J. U. Alvarez-Muñoz, N. Marchand, J. J. Téllez-Guzmán, J. Colmenares-Vazquez, J. Dumon and G. Hasan are with Univ. Grenoble Alpes, GIPSA-Lab, FR-38000 Grenoble, France. Jonatan-Uziel.Alvarez-Munoz@gipsa-lab.grenoble-inp.fr, Nicolas. Marchandeinpg.fr

${ }^{2}$ N. Marchand is with CNRS, GIPSA-Lab, FR-38000 Grenoble, France.

${ }^{3}$ J. F. Guerrero-Castellanos and A.E. López-Luna are with Autonomous University of Puebla (BUAP), Faculty of Electronics, MX-72570 Puebla, Mexico. fguerrero, aluna@ece.buap.mx

${ }^{4}$ S. Durand is with Aix-Marseille University, ISM, FR-13009, Marseille, France. sylvaindeurandchamontin. fr

*This work was supported in part by CONACYT-Mexico, LabEx PERSYVAL-Lab (ANR-11-LABX-0025) and Equipex ROBOTEX (ANR-10-EQPX-44-01).
}

estimate the inertia of the payload and adapt to it in order to improve tracking performance.

Numerous approaches have been proposed to deal with such a problem. In [12], a Newton-Euler approach is used to model and control a manipulator based quadrotor. In [7], a Lyapunov based model Reference Adaptive Control is used to stabilize a quadrotor with multi degree of freedom (DOF) manipulator. However, the stability analysis is carried out with a linear approach and only the dynamics of the quadrotor (considered as a rigid body) was concerned due to the complexity of the system.

In [4], indoor experiments are performed with a quadrotor equipped with a gripper, where an IR camera is used to grip an object with LED placed on it. But this contribution is limited to the use of a 1-DOF gripper, which reduces the precision of manipulation.

In [8], Cartesian impedance control and redundancy are studied using Euler-Lagrange formulation.

The contribution of the present paper is centered on a nonlinear control design and a formal stability analysis for the asymptotical stabilization of a nano-hexacopter carrying a manipulator arm, which is seldom tackled in the literature reviewed. Contrary to the mentioned research, the design of the attitude control law uses the quaternion parametrization, which avoid the presence of singularities. With quaternion parametrization one proposes a constructive control law for the attitude and position stabilization. Firstly, the design of an almost globally asymptotically control law for attitude stabilization which take into account the arm motion effects, is carried out. After that, a globally asymptotically nonlinear controller for the translational dynamics is proposed. In general the control law is based in the usage of nested and sum of saturation functions in order to take into account the actuators limitation. Real-time experimental results validate the proposed strategy.

The paper is structured as follows. In section II, the attitude model of the hexacopter with the manipulator arm is given. Then, the attitude control design is formulated in section III. Section IV is devoted to the design of the position controller. Section V presents experimental results. Finally, some conclusions are presented in section VI.

\section{SYSTEM MODELING}

\section{A. Unit quaternion and attitude kinematics}

Consider two orthogonal right-handed coordinate frames: the body coordinate frame, $B\left(x_{b}, y_{b}, z_{b}\right)$, located at the center of mass of the rigid body and the inertial 
coordinate frame, $N\left(x_{n}, y_{n}, z_{n}\right)$, located at some point in the space (for instance, the earth NED frame). The rotation of the body frame $B$ with respect to the fixed frame $N$ is represented by the attitude matrix $R \in S O(3)=\left\{R \in \mathbb{R}^{3 \times 3}: R^{T} R=I\right.$, det $\left.R=1\right\}$.

The cross product between two vectors $\xi, \varrho \in \mathbb{R}^{3}$ is represented by a matrix multiplication $\left[\xi^{\times}\right] \varrho=\xi \times \varrho$, where $\left[\xi^{\times}\right]$is the well known skew-symmetric matrix.

The $n$-dimensional unit sphere embedded in $\mathbb{R}^{n+1}$ is denoted as $\mathbb{S}^{n}=\left\{x \in \mathbb{R}^{n+1}: x^{T} x=1\right\}$. Members of $S O(3)$ are often parameterized in terms of a rotation $\beta \in \mathbb{R}$ about a fixed axis $e_{v} \in \mathbb{S}^{2}$ by the map $\mathcal{U}: \mathbb{R} \times \mathbb{S}^{2} \rightarrow S O(3)$ defined as

$$
\mathcal{U}\left(\beta, e_{v}\right):=I_{3}+\sin (\beta)\left[e_{v}^{\times}\right]+(1-\cos (\beta))\left[e_{v}^{\times}\right]^{2}
$$

Hence, a unit quaternion, $q \in \mathbb{S}^{3}$, is defined as

$$
q:=\left(\begin{array}{c}
\cos \frac{\beta}{2} \\
e_{v} \sin \frac{\beta}{2}
\end{array}\right)=\left(\begin{array}{c}
q_{0} \\
q_{v}
\end{array}\right) \in \mathbb{S}^{3}
$$

where $q_{v}=\left(\begin{array}{lll}q_{1} & q_{2} & q_{3}\end{array}\right)^{T} \in \mathbb{R}^{3}$ and $q_{0} \in \mathbb{R}$ are known as the vector and scalar parts of the quaternion respectively. The quaternion $q$ represents an element of $S O(3)$ through the map $\mathcal{R}: \mathbb{S}^{3} \rightarrow S O(3)$ defined as

$$
\mathcal{R}:=I_{3}+2 q_{0}\left[q_{v}^{\times}\right]+2\left[q_{v}^{\times}\right]^{2}
$$

Remark 2.1: $R=\mathcal{R}(q)=\mathcal{R}(-q)$ for each $q \in \mathbb{S}^{3}$, i.e. even quaternions $q$ and $-q$ represent the same physical attitude.

Denoting by $\vec{\omega}=\left(\begin{array}{lll}\omega_{1} & \omega_{2} & \omega_{3}\end{array}\right)^{T}$ the angular velocity vector of the body coordinate frame, $B$ relative to the inertial coordinate frame $N$ expressed in $B$, the kinematics equation is given by

$$
\left(\begin{array}{c}
\dot{q}_{0} \\
\dot{q}_{v}
\end{array}\right)=\frac{1}{2}\left(\begin{array}{c}
-q_{v}^{T} \\
I_{3} q_{0}+\left[q_{v}^{\times}\right]
\end{array}\right) \vec{\omega}=\frac{1}{2} \Xi(q) \vec{\omega}
$$

The attitude error is used to quantify mismatch between two attitudes. If $q$ defines the current attitude quaternion and $q_{d}$ the desired quaternion, i.e. the desired orientation, then the error quaternion that represents the attitude error between the current orientation and the desired one is given by

$$
q_{e}:=q_{d}^{-1} \otimes q=\left(q_{e_{0}} q_{e_{v}}^{T}\right)^{T}
$$

where $q^{-1}$ is the complementary rotation of the quaternion $q$ which is given by $q^{-1}:=\left(q_{0}-q_{v}^{T}\right)^{T}$ and $\otimes$ denotes the quaternion multiplication [13].

\section{B. Model of a hexacopter carrying a manipulator arm}

The attitude dynamics and kinematics for the hexacopter have been reported in many works e.g. [5], [6]. In these works it is considered that the hexacopter mass distribution is symmetric. However, the mass distribution of a hexacopter with a manipulator is no longer symmetrical and varies with the movement of the arm. Consider a hexacopter with a manipulator arm with $n$ links attached to its lower part. If the dynamics of the arm is neglected, the attitude kinematics and dynamics is given by

$$
\begin{gathered}
\left(\begin{array}{c}
\dot{q}_{0} \\
\dot{q}_{v}
\end{array}\right)=\frac{1}{2} \Xi(q) \vec{\omega} \\
J \dot{\vec{\omega}}=-\vec{\omega}^{\times} J \vec{\omega}+\Gamma_{T}
\end{gathered}
$$

where $J \in \mathbb{R}^{3 \times 3}$ is the symmetric positive definite constant inertial matrix of the rigid body expressed in the body frame $B$ and $\Gamma_{T} \in \mathbb{R}^{3}$ is the vector of applied torques. $\Gamma_{T}$ depends on the control couples generated by the actuators, the gyroscopic couples, the gravity gradient or, as in the case of the present work, the couple generated by the movement of a robot manipulator placed under the body. Here, only the control couples, gyroscopic couples and the couple generated by the manipulator is considered in the control design. Consequently,

$$
\Gamma_{T}=\Gamma+\Gamma_{a r m}+\Gamma_{G}
$$

where $\Gamma$ and $\Gamma_{G}$ will be described in the section II-C. On the other hand, the vector $\Gamma_{a r m}$ is the torque generated by the total propulsive force being applied at the hexacopter geometric center which is displaced from the center of mass, [14]. This torque can be computed by

$$
\Gamma_{a r m}=m_{a} g \zeta_{c} \times \mathcal{R}(q) e_{3}
$$

where $m_{a}=\sum_{i=1}^{n} m_{m i}+m_{l}$ is the total mass of the manipulator and the load, $m_{m i}$ is the mass of each link of the manipulator and $m_{l}$ is the mass of the load, $g$ is the gravity, $\zeta_{c}=\left(\zeta_{c x} \zeta_{c y} \zeta_{c z}\right)^{T} \in \mathbb{R}^{3}$ is the position of center of mass of the hexacopter with respect to the pivot point in the body frame, $\mathcal{R}(q)$ is the rotation matrix and $e_{3}=\left(\begin{array}{lll}0 & 0 & 1\end{array}\right)^{T}$. Then, the center of mass can be computed by

$$
\zeta_{c}=\frac{1}{m_{a}}\left[\sum_{i=1}^{n} m_{i} \varrho_{i}+m_{l} \varrho_{l}\right]
$$

where $\varrho_{i}$ and $\varrho_{l}$ are the position vector of each link of the manipulator and the load, respectively, both with respect to the reference body frame given by the hexacopter.

In our case, let's consider the scheme in Fig. 1, which shows a two-degrees of freedom manipulator arm then, the corresponding $\varrho_{i}$, where $i=\{1,2\}$, is given by

$$
\begin{aligned}
\varrho_{1} & =\left[\begin{array}{ccc}
0 & 0 & -l_{c 1}
\end{array}\right]^{T} \\
\varrho_{2} & =\left[\begin{array}{ll}
l_{c 2} \sin \theta_{a 2} \cos \theta_{a 1} & l_{c 2} \sin \theta_{a 2} \sin \theta_{a 1} \\
& -\left(l_{1}+l_{c 2}\right) \cos \theta_{a 2}
\end{array}\right]^{T} \\
\varrho_{l} & =\left[\begin{array}{ll}
l_{2} \sin \left(\theta_{a 2}\right) \cos \theta_{a 1} & l_{2} \sin \left(\theta_{a 2}\right) \sin \theta_{a 1} \\
& -\left(l_{1}+l_{2}\right) \cos \left(\theta_{a 2}\right)
\end{array}\right]^{T}
\end{aligned}
$$

where $l_{c 1}$ and $l_{c 2}$ are the distances from the respective joint axes to the center of mass of each link, $l_{1}$ and $l_{2}$ are the total length of the link, and $\theta_{a i}$ measures the angular displacement from $z$ and $x$ axes. 


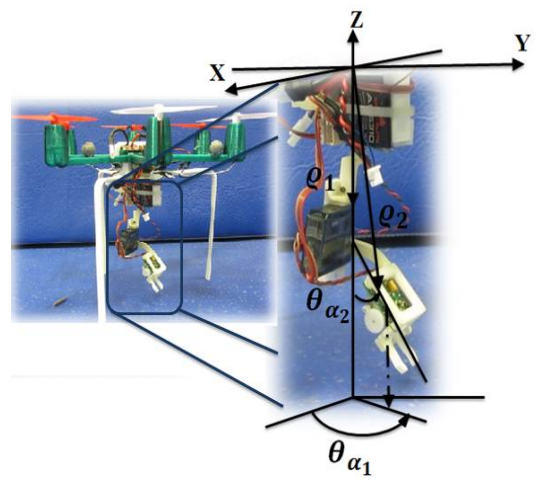

Fig. 1. Manipulator arm with two degrees of freedom.

\section{Actuator model}

The collective input (or throttle input) is the sum of the thrusts of each rotor $f_{1}, f_{2}, f_{3}, f_{4}, f_{5}, f_{6}$. Therefore, the reactive couple $Q_{j}$ generated in the free air by rotor $j$ due to the motor drag and the total thrust $T$ produced by the six rotors can be, respectively, approximated by

$$
\begin{gathered}
Q_{j}=k s_{j}^{2} \\
T=\sum_{j=1}^{6} f_{j}=b \sum_{j=1}^{6} s_{j}^{2}
\end{gathered}
$$

where $s_{j}$ represents the rotational speed of rotor $j . k>0$ and $b>0$ are two parameters depending on the density of air, the radius, the shape, the pitch angle of the blade and other factors [6]. The vector of gyroscopic couples $\Gamma_{G}$ is given by

$$
\Gamma_{G}=\sum_{j=1}^{6} J_{r}\left(\vec{\omega} \times \vec{z}_{b}\right)(-1)^{j+1} s_{j}
$$

where $J_{r}$ is the inertia of the so-called rotor (composed of the motor rotor itself with the gears). The components of the control torque $\Gamma \in \mathbb{R}^{3}$ generated by the rotors are given by $\Gamma=\left[\begin{array}{lll}\Gamma_{1} & \Gamma_{2} & \Gamma_{3}\end{array}\right]^{T}$, with

$$
\begin{gathered}
\Gamma_{1}=d \frac{\sqrt{3}}{2} b\left(s_{2}^{2}+s_{3}^{2}-s_{5}^{2}-s_{6}^{2}\right) \\
\Gamma_{2}=\frac{d b}{2}\left(2 s_{1}^{2}+s_{2}^{2}-s_{3}^{2}-2 s_{4}^{2}-s_{5}^{2}+s_{6}^{2}\right) \\
\Gamma_{3}=k\left(s_{1}^{2}+s_{2}^{2}+s_{3}^{2}+s_{4}^{2}+s_{5}^{2}+s_{6}^{2}\right)
\end{gathered}
$$

where $d$ is the distance between the rotor and the center of gravity of the hexacopter

\section{ATTITUDE CONTROL DESIGN}

\section{A. Problem statement}

The objective is to design a control law which drives the hexacopter to attitude stabilization under the torques and moments exerted to this from the movement of a manipulator arm attached to its lower part. In other words, let $q_{d}$ denote the constant hexacopter stabilization orientation, then the control objective is described by the following asymptotic conditions

$$
q \rightarrow\left[\begin{array}{llll} 
\pm 1 & 0 & 0 & 0
\end{array}\right]^{T}, \vec{\omega} \rightarrow 0 \text { as } t \rightarrow \infty
$$

Furthermore, it is known that actuator saturation reduces the benefits of the feedback. Then, besides the asymptotic stability, the control law also takes into account the physical constraints of the control system, in order to apply only feasible control signals to the actuators.

\section{B. Attitude control with manipulator arm}

In this subsection, a control law that stabilizes the system described by (6) and (7) is proposed. The goal is to design a control torque that is bounded.

Definition 3.1: Given a positive constant $M$, a continuous, nondecreasing function $\sigma_{M}: \mathbb{R} \rightarrow \mathbb{R}$ is defined by

$$
\begin{aligned}
& (1) \sigma_{M}=s \text { if }|s|<M ; \\
& (2) \sigma_{M}=\operatorname{sign}(s) M \text { elsewhere. }
\end{aligned}
$$

Note that the components of $\Gamma_{a r m_{i}}$ are always bounded, i.e. $\left|\Gamma_{a r m_{i}}\right|<\delta_{i}$. Then, one has the following result.

Theorem 3.2: Consider a rigid body rotational dynamics described by (6) and (7) with the following bounded control inputs $\Gamma=\left(\begin{array}{lll}\Gamma_{1} & \Gamma_{2} & \Gamma_{3}\end{array}\right)^{T}$ such that

$$
\Gamma_{i}=-\sigma_{M_{i 2}}\left(\Gamma_{a r m_{i}}+\sigma_{M_{i 1}}\left(\lambda_{i}\left[\omega_{i}+\rho_{i} q_{i}\right]\right)\right)
$$

with $i \in\{1,2,3\}$ and where $\sigma_{M_{i 1}}$ and $\sigma_{M_{i 2}}$ are saturation functions. Assuming $\delta_{i}<M_{i 2}-M_{i 1}$ and $M_{i 1} \geq 3 \lambda_{i} \rho_{i} . \lambda_{i}$ and $\rho_{i}$ are positive parameters. Then the inputs (20) asymptotically stabilize the rigid body to the origin $\left(\begin{array}{lll}1 & 0^{T} & 0^{T}\end{array}\right)^{T}$ (i.e. $q_{0}=1, q_{v}=0$ and $\vec{\omega}=0$ ) with a domain of attraction equal to $\mathbb{S}^{3} \times \mathbb{R}^{3} \backslash\left(\begin{array}{lll}-1 & 0^{T} & 0^{T}\end{array}\right)^{T}$.

Due to space constraints, the proof of this Theorem is not presented here.

Remark 3.3: Note that the stability analysis has been carried out considering the asymptotic condition $q \rightarrow q_{d}=$ $\left[\begin{array}{llll} \pm 1 & 0 & 0 & 0\end{array}\right]^{T}$. In the case where the asymptotic condition $q \rightarrow q_{d}$ with $q_{d} \neq\left[\begin{array}{llll} \pm 1 & 0 & 0 & 0\end{array}\right]^{T}$ is considered, the control law applied will be

$$
\Gamma_{i}=-\sigma_{M_{i 2}}\left(\Gamma_{a r m_{i}}+\sigma_{M_{i 1}}\left(\lambda_{i}\left[\omega_{i}+\rho_{i} q_{e_{i}}\right]\right)\right)
$$

where $q_{e}$ represents the attitude error between the current orientation and the desired one.

\section{POSITION CONTROL DESIGN}

\section{A. Problem statement}

The objective is to design a control law which stabilizes the hexacopter to a desired position, having the attitude stabilization problem solved. In other words, once the control law has stabilized the attitude of the system, $\lim _{t \rightarrow \infty}(q, \vec{\omega})=$ $\left(q_{d}, \overrightarrow{0}\right)$, the position control law should stabilize the hexacopter in a desired position, $\lim _{t \rightarrow \infty}(\vec{p}, \vec{v})=\left(\vec{p}_{d}, \overrightarrow{0}\right)$, even under the disturbances from the manipulator arm. 


\section{B. Position stabilization strategy}

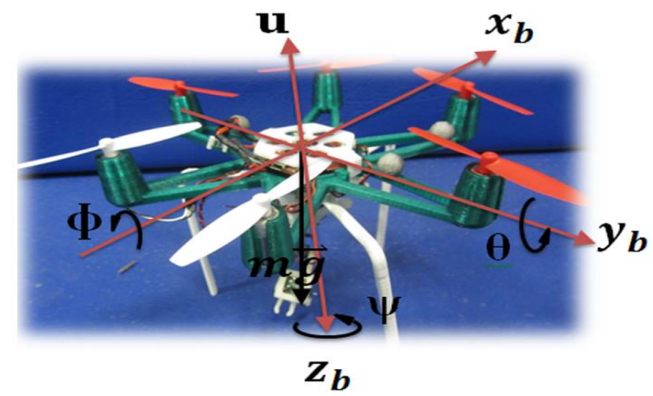

Fig. 2. Schematic configuration of the hexacopter carrying a manipulator arm.

The schematic representation of a hexacopter carrying a manipulator arm can be seen in Fig. 2, where the body reference frame $B\left(x_{b}, y_{b}, z_{b}\right)$, the force $u$ (thrust) and the weight vector $m \vec{g}$ are depicted. The dynamics of the whole system is obtained with the Newton-Euler formalism and the kinematics is represented using the quaternions formalism:

$$
\begin{aligned}
& \Sigma_{T}:\left\{\begin{array}{l}
\dot{\vec{p}}=\vec{v} \\
m_{T} \dot{\vec{v}}=-m_{T} \vec{g}+R\left(\begin{array}{l}
0 \\
0 \\
u
\end{array}\right)
\end{array}\right. \\
& \Sigma_{O}:\left\{\begin{array}{l}
\dot{q}=\frac{1}{2} \Xi(q) \vec{\omega} \\
J \dot{\vec{\omega}}=-\vec{\omega} \times J \vec{\omega}+\Gamma_{T}
\end{array}\right.
\end{aligned}
$$

where $\vec{p}$ and $\vec{v}$ are linear position and velocity vectors, $m_{T}$ is the total mass of the system (hexacopter and manipulator), $\vec{g}$ is the gravity, $R$ is the rotation matrix, given in (3).

Note that the rotation matrix $R$ can also be given in function of the Euler angles [3].

Equations (22) and (23) show that the translational dynamics (22) depends on the attitude (23), but not the opposite. This property will be used to design the control law. Now, assume that using the control law (20) one can stabilize the yaw dynamics, that is $\psi=0$, then after a sufficiently long time, system (22) becomes:

$$
\begin{aligned}
& \left(\begin{array}{c}
\dot{p}_{x} \\
\dot{p}_{y} \\
\dot{p}_{z}
\end{array}\right)=\left(\begin{array}{c}
v_{x} \\
v_{y} \\
v_{z}
\end{array}\right), \\
& \left(\begin{array}{c}
\dot{v}_{x} \\
\dot{v}_{y} \\
\dot{v}_{z}
\end{array}\right)=\left(\begin{array}{c}
-\frac{u}{m_{T}} \sin \theta \\
\frac{u}{m_{T}} \sin \phi \cos \theta \\
\frac{u}{m_{T}} \cos \phi \cos \theta-g
\end{array}\right)
\end{aligned}
$$

With an appropriate choice of the target configuration, it will be possible to transform (24)-(25) into three independent linear triple integrators. For this, take

$$
\begin{aligned}
\phi_{d} & :=\arctan \left(\frac{r_{2}}{r_{3}+g}\right), \\
\theta_{d} & :=\arcsin \left(\frac{-r_{1}}{\sqrt{r_{1}^{2}+r_{2}^{2}+\left(r_{3}+g\right)^{2}}}\right)
\end{aligned}
$$

where $r_{1}, r_{2}$ and $r_{3}$ will be defined after. Then, choose as positive thrust the input control

$$
u=m_{T} \sqrt{r_{1}^{2}+r_{2}^{2}+\left(r_{3}+g\right)^{2}}
$$

Let the state be $p=\left(p_{1}, p_{2}, p_{3}, p_{4}, p_{5}, p_{6}, p_{7}, p_{8}, p_{9}\right)=$ $\left(\int p_{x}, p_{x}, v_{x}, \int p_{y}, p_{y}, v_{y}, \int p_{z}, p_{z}, v_{z}\right)$, then (24)-(25) becomes:

$$
\Sigma_{x}:\left\{\begin{array}{l}
\dot{p}_{1}=p_{2} \\
\dot{p}_{2}=p_{3} \\
\dot{p}_{3}=r_{1}
\end{array} \quad \Sigma_{y}:\left\{\begin{array}{l}
\dot{p}_{4}=p_{5} \\
\dot{p}_{5}=p_{6} \\
\dot{p}_{6}=r_{2}
\end{array} \quad \Sigma_{z}:\left\{\begin{array}{l}
\dot{p}_{7}=p_{8} \\
\dot{p}_{8}=p_{9} \\
\dot{p}_{9}=r_{3}
\end{array}\right.\right.\right.
$$

Note that $u$ will be always positive, and $u \geq m g$, in order to compensate the system's weight.

Since the chains of integrators given in (28) have the same form, a control law can be proposed as in [3], and can be established by the next theorem:

Theorem 4.1: Consider the hexacopter translational dynamics expressed in (24-25). Then, the thrust input $u$ given by (13) with $r_{1}, r_{2}, r_{3}$ as in (29), where $\sigma_{M_{1}}(\cdot)$ is defined in (19) with $M_{1}=1$ and $\varsigma_{i}$ are given by (30), where $a_{(1,2,3)}, b_{(1,2,3)}, c_{(1,2,3)}>0$ tuning parameters.

$$
\begin{aligned}
& r_{1}:=-\varsigma_{1}\left\{a_{3} \sigma_{M 1}\left[\frac{1}{\varsigma_{1}}\left(a_{2} p_{1}+p_{2}+p_{3}\right)\right]\right. \\
&+\left.a_{2} \sigma_{M 1}\left[\frac{1}{\varsigma_{1}}\left(a_{1} p_{2}+p_{3}\right)\right]+a_{1} \sigma_{M 1}\left[\frac{1}{\varsigma_{1}}\left(p_{3}\right)\right]\right\}, \\
& r_{2}:=-\varsigma_{2}\left\{b_{3} \sigma_{M 1}\left[\frac{1}{\varsigma_{1}}\left(b_{2} p_{4}+p_{5}+p_{6}\right)\right]\right. \\
&\left.+b_{2} \sigma_{M 1}\left[\frac{1}{\varsigma_{2}}\left(b_{1} p_{5}+p_{6}\right)\right]+b_{1} \sigma_{M 1}\left[\frac{1}{\varsigma_{2}}\left(p_{6}\right)\right]\right\}, \\
& r_{3}:=-\varsigma_{3}\left\{c_{3} \sigma_{M 1}\left[\frac{1}{\varsigma_{1}}\left(c_{2} p_{7}+p_{8}+p_{9}\right)\right]\right. \\
&\left.+c_{2} \sigma_{M 1}\left[\frac{1}{\varsigma_{3}}\left(c_{1} p_{8}+p_{9}\right)\right]+c_{1} \sigma_{M 1}\left[\frac{1}{\varsigma_{3}}\left(p_{9}\right)\right]\right\} \\
& \varsigma_{1}=\bar{r}_{1} /\left(a_{1}+a_{2}+a_{3}\right), \\
& \varsigma_{2}=\bar{r}_{2} /\left(b_{1}+b_{2}+b_{3}\right), \\
& \varsigma_{3}=\bar{r}_{3} /\left(c_{1}+c_{2}+c_{3}\right)
\end{aligned}
$$

The proof of this Theorem is not presented here, but it can be derived from the seminal work of [9], [10] and [11].

Remark 4.2: In the above Theorem, the stabilization goal is the origin. When the asymptotic condition is different, the variables $p_{2}, p_{5}, p_{8}$ should be replaced in the control law (29) by $e_{1}=p_{2}-p_{x}^{d}, e_{2}=p_{5}-p_{y}^{d}, e_{3}=p_{8}-p_{z}^{d}$, respectively. Here, $p_{x}^{d}, p_{y}^{d}, p_{z}^{d}$ represent the desired position in the space.

\section{EXPERIMENTAL SETUP}

\section{A. Hardware setup}

The aerial system consists on the Flexbot hexacopter set [15], which structure was modified. The arm length is $7.1 \mathrm{~cm}$, the weight is $70 \mathrm{~g}$ and the carrying capacity $32 \mathrm{~g}$. On the 
other hand, a manipulator arm was designed and built for this project, whose length links are $5 \mathrm{~cm}$ for the first one and $3 \mathrm{~cm}$ for the second one and its weight $28 \mathrm{~g}$. The total weight of the system is $98 \mathrm{~g}$ and its carrying capacity is about $4 \mathrm{~g}$. The attitude control law (20) for the hexacopter was programmed in a Microwii Copter board. The Vicon Tracker system [16], MATLAB/Simulink and the XPC target toolbox [17] are used to implement the position control. Fig. 3 shows an overview of the whole hardware architecture.

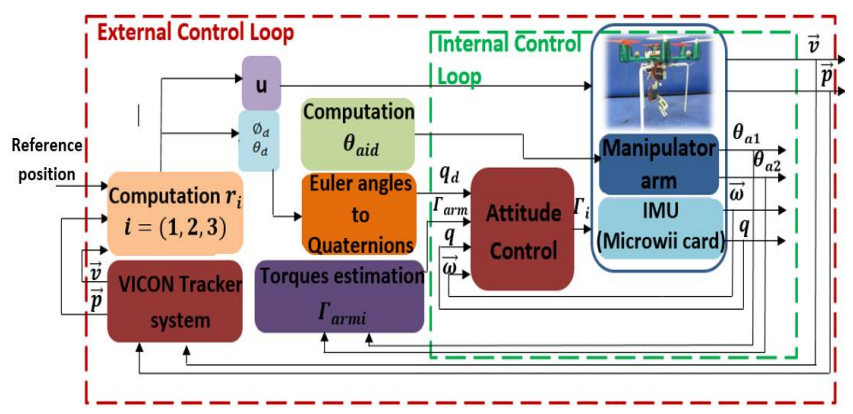

Fig. 3. Block diagram of the system.

\section{B. Experimental scenario}

Two experiments are performed in order to compare the performance of the proposed control with and without taking into account the torque generated by the manipulator. The parameters of the control law are selected according to the characteristics of the actuators and those of the hexacopter presented previously. The initial position and attitude of the hexarotor for both experiments are close to zero.

The scenario for the experiments is divided in two parts. For the first $10 \mathrm{~s}$, the hexacopter is driven to the position $p_{d}=\left(\begin{array}{lll}0 & 0 & 1\end{array}\right)^{T}$. The manipulator arm orientation is initialized at $\theta_{a i}=\left(\begin{array}{ll}0 & 0\end{array}\right)^{T}$ and it does not change during the stabilization. Then, two movements are performed with the manipulator. At time $10 \mathrm{~s}, \theta_{a 1}$ and $\theta_{a 2}$ are oriented at $60^{\circ}$ and $45^{\circ}$ respectively. At time $15 \mathrm{~s}$, the orientations are changed to -60 and $90^{\circ}$ respectively. At time $20 \mathrm{~s}$, the full system returns to its initial position.

In Fig. 4 and Fig. 5, angular position of the manipulator, linear and angular position, linear and angular errors position, average attitude error (Ave), attitude control torques of the hexacopter and estimated torques for the manipulator are depicted. Note that even when the quaternion parametrization is considered, Euler angles are used in order to have a better perspective of the behavior of the system. In the first case, attitude stabilization is reached, but some oscillations are present. Then, the response is improved in Fig. 5 when the estimation of the torque is taken into account, the attitude stabilization is achieved. The study of the position errors and the average value attitude error show that the response of the system is improved when the estimation of the torque from the manipulator is used. In order to calculate the attitude error, $\left\|2 \arccos q_{0}\right\|$ was used, where $\|\cdot\|$ represents the norm value. The average attitude error values obtained from the experiments are Ave $=0.9955,0.7944$, where the lower one is obtained when the proposed method is applied, which also reduced the torque generated from the arm.

\section{CONCLUSIONS}

In this paper, a control law was designed to asymptotically stabilize the attitude and position of a hexacopter carrying a manipulator arm. Moreover, this work has presented a method for aiding the solution through the design of a feedforward term which allows the estimation of the torques exerted by the manipulator. Also, the control law takes into account the actuators saturations. Experimental results show the effectiveness of the proposed control law face to the disturbances coming from the manipulator. Multiple trials and scenarios are carried out in order to statistically determine the effectiveness of the method. Experimental load mass estimation will be pursued as a future work.

\section{REFERENCES}

[1] Mellinger, D.; Shomin, M.; Michael, N.; Kumar, V., "Cooperative Grasping and Transport using Multiple Quadrotors," International Symposium on Distributed Autonomous Systems. Lausanne, Switzerland 2010.

[2] Michael, N.; Fink, J.; Kumar, V., "Cooperative manipulation and transportation with aerial robots," Proceedings of Robotics: Science and Systems, Seattle, USA, June. 2009.

[3] Cruz-José, R.; Guerrero-Castellanos, J.F.; Guerrero-Sánchez, W.F. and Oliveros-Oliveros, J.J. , "Estabilización global de mini naves aéreas tipo VTOL,” In Congreso Nacional de Control Automático. Campeche, México, 2012.

[4] Ghadiok, Vaibhav; Goldin, Jeremy; Wei Ren, "Autonomous indoor aerial gripping using a quadrotor," Intelligent Robots and Systems (IROS), 2011 IEEE/RSJ International Conference on , vol., no., pp.4645,4651, 25-30 Sept. 2011

[5] Guerrero-Castellanos, J.F.; Marchand, N.; Hably, N.; Lesecq, S.; Delamare, J., "Bounded attitude control of rigid bodies: Real time experimentation to a quadrotor mini-helicopter," Control Engineering Practice, vol.19, no., pp.790,797, 2011.

[6] Alaimo, A.; Artale, V.; Milazzo, C.; Ricciardello, A.; Trefiletti, L., "Mathematical modeling and control of a hexacopter," Unmanned Aircraft Systems (ICUAS), 2013 International Conference on , vol., no., pp.1043,1050, 28-31 May 2013.

[7] Orsag, M.; Korpela, C.; Bogdan, S.; Oh, P., "Lyapunov based model reference adaptive control for aerial manipulation," Unmanned Aircraft Systems (ICUAS), 2013 International Conference on , vol., no., pp.966,973, 28-31 May 2013.

[8] Lippiello, V.; Ruggiero, F., "Exploiting redundancy in Cartesian impedance control of UAVs equipped with a robotic arm," Intelligent Robots and Systems (IROS), 2012 IEEE/RSJ International Conference on , vol., no., pp.3768,3773, 7-12 Oct. 2012.

[9] Marchand, N.; Hably, A., "Global stabilization of multiple integrators with bounded controls," Automatica, vol.41, pp.2147,2152, 2005.

[10] Teel, A. R., "Global stabilization and restricted tracking for multiple integrators with bounded controls," Systems and Control Letters, vol.18, no.3, pp.165,171, 1992.

[11] Johnson, E. N.; Kannan, S. K., "Nested saturation with guaranteed real poles," Proceedings of the American Control Confernce in, vol.1, pp.497,502, 2003.

[12] Orsag, M.; Korpela, C.; Oh, P., "Modeling and Control of MMUAV: Mobile Manipulating Unmanned Aerial Vehicle," Journal of Intel Robot Sys, pp.227,240. 2013.

[13] Shuster, M., D., "A survey of attitude representations," The Journal of the Astronautical Sciences, pp.439,517. October-December. 1993.

[14] Sangbum, C., "Dynamics and control of underactuated multibody spacecraft," Dissertations And Theses. University of Michigan. 2002.

[15] Flexbot.cc, 'Flexbot - Design, Make and Share', 2014. [Online]. Available: http://www.flexbot.cc/.

[16] Vicon.com, 'Vicon - Systems', 2015. [Online]. Available: http://www.vicon.com/System/TSeries.

[17] MATLAB version 8.5.0. Natick, Massachusetts: The MathWorks Inc., 2012. 

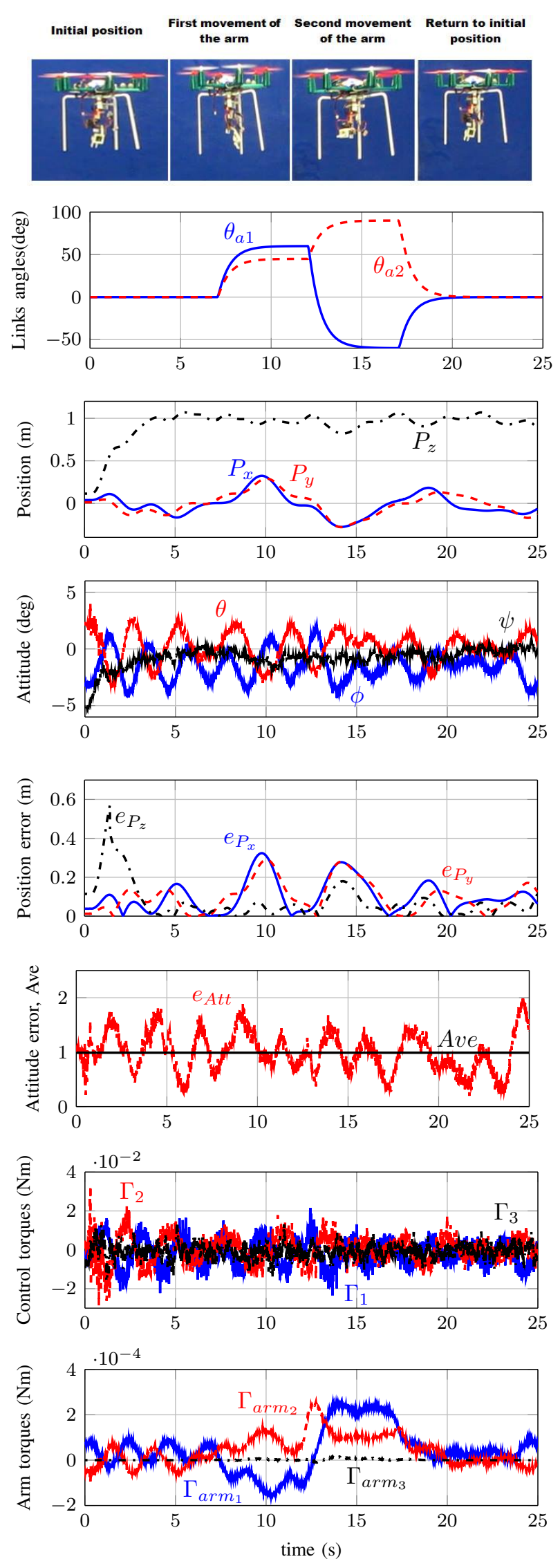

Fig. 4. Experimental results when the torque is not taken into account.
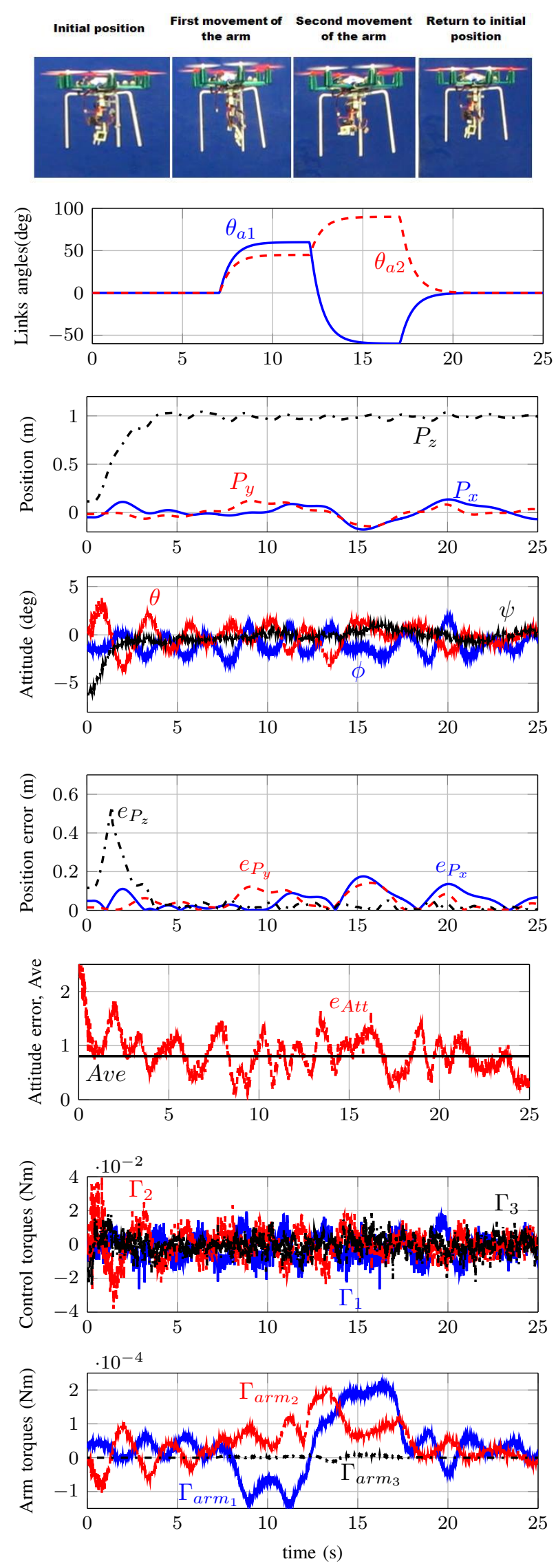

Fig. 5. Experimental results when the torque is taken into account. 